Onkologe 2013 · 19:178-179

DOI 10.1007/s00761-012-2397-5

Online publiziert: 2. März 2013

(c) Springer-Verlag Berlin Heidelberg 2013

A.-L. Grosu ${ }^{1} \cdot$ M. Fassnacht ${ }^{2} \cdot$ M. Bamberg ${ }^{3}$

${ }^{1}$ Klinik für Strahlenheilkunde, Universität Freiburg

${ }^{2}$ Medizinische Klinik und Poliklinik IV, Klinikum der Universität München

${ }^{3}$ Universitätsklinikum Tübingen

\title{
Endokrine und neuroendokrine Tumoren
}

Das aktuelle Heft präsentiert neue diagnostische und therapeutische Möglichkeiten in der Behandlung endokriner und neuroendokriner Tumoren: Phäochromozytome, Nebennierenkarzinome, gastroenteropankreatische neuroendokrine Tumoren und medulläre Schilddrüsenkarzinome.

Die komplexe Gruppe der endokrinen/ neuroendokrinen Tumoren fasst maligne und benigne Geschwülste zusammen, die ihren Ursprung in den endokrinen/ neuroendokrinen Zellen haben. Die Produktion verschiedener Hormone ist die wichtigste gemeinsame Eigenschaft der jeweiligen Ursprungszellen. Darüber hinaus sind diese Zellen durch ultrastrukturelle Gemeinsamkeiten charakterisiert. Lokalisation, funktionelle Eigenschaften und sogar embryologische Herkunft sind sehr unterschiedlich, sodass das diagnostische und therapeutische Bild dieser Tumoren komplex und nach Ursprung und Lokalisation verschieden ist.

Die ersten endokrinen Zellen wurden im Darm von Heidenhain (1870) und Kulchitsky (1897) beschrieben. Der Österreicher Friedrich Feyrter, der als Begründer der Neuroendokrinologie gilt und nach dem Zweiten Weltkrieg an der Georg-August-Universität Göttingen unterrichtet hatte, beschrieb in den 30er Jahren ,helle" epitheliale Zellen, die diffus in verschiedenen Geweben verteilt sind. Masson sprach 1914 über Argentaffin-positive Zellen im Darm und Hamperl (1932) beschrieb die argentaffinen Zellen ebenfalls. Froelich berichtete 1938 über „,helle"Zellen der Bronchialschleimhaut und ihre Beziehung zum Problem der Chemorezeptoren. 40 Jahre später charakte- risierte Pearse die Tumoren als „APUDome“, d. h. Tumoren, deren Ursprung im APUD- System (,amin precursor uptake and decarboxilation system") liegen. Zellen mit APUD-Charakteristika werden heute neuroendokrine Zellen genannt.

\section{》) Die Häufigkeit einzelner endokriner/neuroendokriner Tumoren hat in den letzten Jahren zugenommen}

Endokrine/neuroendokrine Tumoren sind selten; dennoch gibt es Indizien, dass die Häufigkeit einzelner dieser Tumoren in den letzten Jahren klar zugenommen hat.Vor allem aufgrund der variablen Hormonproduktion ist das klinische Bild häufig komplex und verlangt eine intensive interdisziplinäre Zusammenarbeit (unter anderem Endokrinologen, internistische Onkologen, Gastroenterologen, Radiologen, Nuklearmediziner, Pathologen, Chirurgen und Strahlentherapeuten). Erfreulicherweise gab es in den letzten Jahren bei den vorgestellten Tumoren teilweise deutliche Fortschritte und Veränderungen der diagnostischen und therapeutischen Standards, die in diesem Heft zusammenfassend präsentiert werden. Wir hoffen, dem Leser damit einen aktuellen Überblick über diesen vielseitigen Bereich der Onkologie geben zu können. Da bei vielen der behandelten Tumorentitäten aktuell klinische Studien (mehrere in Phase III) laufen, ist damit zu rechnen, dass sich die Therapie in den nächsten Jahren weiter verbessert. Aus diesem Grund empfehlen wir grund- sätzlich, mit einem jeweils spezialisierten Zentrum Kontakt aufzunehmen.

Für die Herausgeber des Schwerpunktthemas

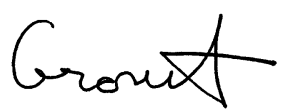

\section{A.-L. Grosu}

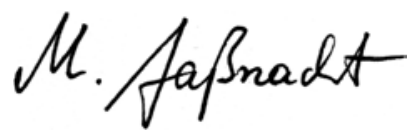

M. Fassnacht

Für die Herausgeber<smiles>C=CC</smiles>

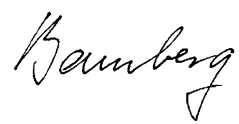

M. Bamberg

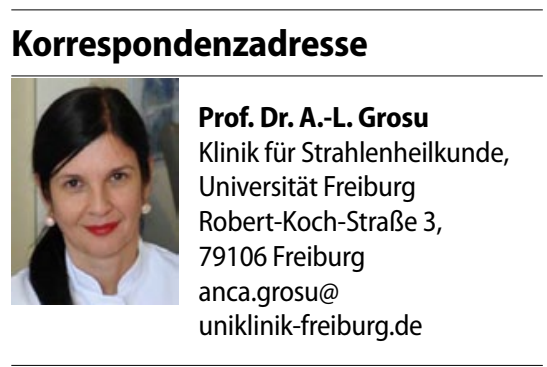


Hier steht eine Anzeige.

黛 Springer 